\title{
C. elegans GLA-3 is a novel component of the MAP kinase MPK-1 signaling pathway required for germ cell survival
}

\author{
Ekaterini A. Kritikou, ${ }^{1,5}$ Stuart Milstein, ${ }^{3,5}$ Pierre-Olivier Vidalain, ${ }^{3,6}$ Guillaume Lettre, ${ }^{1}$ \\ Erica Bogan, ${ }^{1,2}$ Kimon Doukoumetzidis, ${ }^{1,2}$ Phillip Gray, ${ }^{4}$ Thomas G. Chappell, ${ }^{4}$ Marc Vidal, $^{3}$ and \\ Michael O. Hengartner ${ }^{1,7}$ \\ ${ }^{1}$ Institute of Molecular Biology, University of Zurich, 8057 Zurich, Switzerland; ${ }^{2}$ Ph.D. Program in Molecular Life Sciences, \\ University of Zurich, 8057 Zurich, Switzerland; ${ }^{3}$ Center for Cancer Systems Biology (CCSB) and Department of Cancer \\ Biology, Dana Farber Cancer Institute and Department of Genetics, Harvard Medical School, Boston, Massachusetts 02115, \\ USA; ${ }^{4}$ Invitrogen Corporation, Carlsbad, California 92008, USA
}

\begin{abstract}
During oocyte development in Caenorhabditis elegans, approximately half of all developing germ cells undergo apoptosis. While this process is evolutionarily conserved from worms to humans, the regulators of germ cell death are still largely unknown. In a genetic screen for novel genes involved in germline apoptosis in Caenorhabditis elegans, we identified and cloned gla-3. Loss of gla-3 function results in increased germline apoptosis and reduced brood size due to defective pachytene exit from meiosis I. gla-3 encodes a TIS11-like zinc-finger-containing protein that is expressed in the germline, from the L4 larval stage to adulthood.

Biochemical evidence and genetic epistasis analysis revealed that GLA-3 participates in the MAPK signaling cascade and directly interacts with the $C$. elegans MAPK MPK-1, an essential meiotic regulator. Our results show that GLA-3 is a new component of the MAPK cascade that controls meiotic progression and apoptosis in the C. elegans germline and functions as a negative regulator of the MAPK signaling pathway during vulval development and in muscle cells.
\end{abstract}

[Keywords: Apoptosis; GLA-3; MAPK signaling; germline development; C. elegans]

Supplemental material is available at http://www.genesdev.org.

Received February 24, 2006; revised version accepted June 1, 2006.

Germ stem cells are the precursors to all subsequent generations in a species, and, therefore, germ cell formation is tightly monitored to ensure high-fidelity transfer of the genetic material. Germ cell genome integrity is monitored at several checkpoints, allowing for DNA repair, cell cycle arrest, and apoptosis when required. In mammals, inactivation of genes with checkpoint function frequently results in aberrant cell death and infertility (Lim and Hasty 1996; Bender et al. 2002). Germ cell development is also characterized by either massive waves or low but constant levels of apoptosis that are not caused by genetic defects. For example, $>99.9 \%$ of oocytes undergo apoptosis in response to hormonal changes that occur at several stages during the female life cycle in mammals (Tilly 2001; Kim and Tilly 2004). Apoptosis is also the fate of $\sim 50 \%$ of germ cells undergoing oogenesis in the gonad of Caenorhabditis elegans

\footnotetext{
${ }^{5}$ These authors contributed equally to this work.

${ }^{6}$ Present address: Laboratoire génomique virale et vaccination, CNRS UMR1930, Institut Pasteur de Paris, 28 Rue du Docteur Roux, Paris, 75724 Cedex 15, France.

${ }^{7}$ Corresponding author.

E-MAIL michael.hengartner@molbio.unizh.ch; FAX 41-44-635-6861.

Article is online at http://www.genesdev.org/cgi/doi/10.1101/gad.384506.
}

hermaphrodites. (Gumienny et al. 1999). Characterization of the pathways that regulate germ cell death will contribute to our understanding of the cell suicide decision and might allow for more efficient therapeutic manipulation of the apoptotic program.

C. elegans is a good model to study the signaling cascades involved in the decision between germ cell survival and germ cell death (Hengartner 1997; Gumienny et al. 1999). The adult hermaphrodite gonads consist of two U-shaped tubes that are connected at a common uterus. At the distal end of each gonad, mitotic germ stem cells proliferate in response to the Notch ligand LAG-2. Cells beyond the influence of LAG-2 enter meiosis and progress through the pachytene stage of meiosis I; this transition requires activation of the RAS/MAPK (MAP kinase) signaling cascade (Hubbard and Greenstein 2000; Seydoux and Schedl 2001). Following transition through pachytene, germ cells can either enter diakinesis of meiosis I and differentiate into oocytes or undergo apoptosis. We previously suggested that these cell deaths are the result of a physiological, homeostatic control mechanism that limits the number of germ cells permitted to differentiate into oocytes (Gumienny et al. 1999). 
During C. elegans somatic development, apoptosis is triggered when the pro-apoptotic $\mathrm{BH} 3$-domain-containing protein EGL-1 interacts with the BCL-2 family member CED-9 on the surface of mitochondria. Binding of EGL-1 induces CED-9 to release the sequestered Apaf-1 homolog CED-4, resulting in the formation of a C. elegans apoptosome and activation of the caspase CED-3 (Chen et al. 2000; Yan et al. 2004, 2005; for review, see Lettre and Hengartner 2006). We previously showed that germline apoptosis can be induced by both p53-dependent and p53-independent pathways (Lettre et al. 2004). However, whereas DNA damage-induced germline apoptosis depends on the activation of EGL-1, physiological germline apoptosis occurs normally in the absence of EGL-1, indicating that additional regulatory factors are required to trigger the apoptotic cascade in these cells (Gumienny et al. 1999; Gartner et al. 2000).

In an attempt to identify genes that regulate physiological germ cell apoptosis, we performed a forward genetic screen to isolate mutants with increased levels of germline apoptosis. In this paper, we report the cloning and characterization of gla-3 (germline apoptosis), a gene that encodes a predicted RNA-binding protein of the TIS11 family. Loss of gla-3 function results in increased germ cell apoptosis and severe defects in oocyte differentiation, which lead to reduced brood size. Biochemical analysis revealed that GLA-3 physically interacts with the $C$. elegans MAPK MPK-1, providing a direct link between the apoptotic process and oogenesis. Furthermore, we show that GLA-3 functions as an inhibitor of the MAPK cascade during vulva formation and in muscle cells. Our findings indicate that GLA-3 is a new component of the MAPK signaling cascade and highlight a molecular link between germ cell survival and pachytene progression in the C. elegans germline.

\section{Results}

Loss of gla-3 function results in increased germ cell death

To identify novel genes involved in the regulation of germcell apoptosis, we performed a forward genetic screen to isolate mutations that result in increased germline apoptosis, as described in Materials and Methods. gla-3 was selected for cloning and further characterization based on the severity of its germline apoptosis phenotype. All three gla-3 alleles that we analyzed lop212 and op216, isolated in our screen, and ep312, generously provided by M. Costa, Exelixis) resulted in increased numbers of germ cell corpses that displayed typical apoptotic morphology (Fig. 1A,B). Because gla-3 mutants exhibited normal patterns of somatic cell death (data not shown), we conclude that gla-3 is not a general cell-death regulator.

To investigate whether loss of gla-3 function results in increased germ cell apoptosis rather than decreased engulfment, we analyzed gla-3 mutants carrying the integrated transgene opIs110, which expresses a YFP-tagged version of cytosolic actin. We previously showed that this YFP::ACT-5 reporter is highly enriched around apoptotic germ cells that are in the process of being engulfed (Kinchen et al. 2005). Whereas inactivation of the engulfment gene ced-1 largely abolished YFP::actin staining, most apoptotic germ cells in gla-3(lf); opIs110 (lf, loss of function) animals were surrounded by a YFP:: actin halo, indicating that the cells were being engulfed efficiently (Supplemental Fig. 1). These results support our notion that loss of gla-3 function results in increased germ cell death, rather than decreased cell corpse clearance.

To determine an epistatic relationship between gla-3 and other components of the apoptotic machinery, we generated strains that contain gla-3(op212) and either of the strong loss-of-function mutations ced-4(n1162) or ced-3(n717). In both double mutants, germ cell death was completely abrogated, demonstrating that gla-3(lf)induced cell death is apoptotic in nature and that the components of the apoptotic machinery function downstream of gla-3 (Fig. 1C).

Because germline apoptosis can be mediated by both DNA damage-dependent and -independent mechanisms (Gartner et al. 2000), we carried out epistasis analysis between g1a-3(op212) and genes that are known to be involved in DNA damage responses. hus-1(op244) and clk-2(mn159ts) mutants are characterized by a complete absence of DNA damage-induced apoptosis, but have nearly normal levels of somatic and germline apoptosis (Ahmed et al. 2001; Hofmann et al. 2002). gla-3(op212); clk-2(mn159ts) and hus-1(op244) gla-3(op212) worms had similar levels of germline apoptosis as gla-3(op212) alone, indicating that gla-3-induced apoptosis occurs independently of these DNA damage checkpoint genes (Fig. 1D). Because DNA damage-induced apoptosis is dependent on the p53 homolog cep-1 (Derry et al. 2001; Schumacher et al. 2001), we inactivated gla-3 using RNA-mediated interference (RNAi) in cep-1 (gk138) mutants. Consistent with our previous findings (Lettre et al. 2004), we observed similar levels of germline apoptosis in gla-3(RNAi) cep-1(gk138) and in gla-3(RNAi) alone (Fig. 1E), showing that loss of gla-3 does not activate a DNA damage apoptotic response.

Finally, we tested the ability of gla-3 mutants to respond to exogenous DNA damage. We previously showed that ionizing radiation induces apoptosis of meiotic cells and transient cell cycle arrest of the mitotic germ cell population (Gartner et al. 2000). Both apoptosis and cell cycle responses appeared normal in gla-3(op212) mutants (Fig. 1D; Supplemental Fig. 2, respectively). These data suggest that the DNA damage response pathways are functional in gla-3 mutants, and are consistent with a role of gla-3 in the physiological cell-death pathway.

\section{Identification of mutations in the $\mathrm{C}$. elegans gla-3 gene}

We genetically mapped gla-3(op212) to a 0.36-cM interval, on the right arm of Chromosome I, between the single nucleotide polymorphism markers pk1058 at 2.31 $\mathrm{cM}$ and $o p P 106$ at $2.67 \mathrm{cM}$, as described in Materials and Methods. We inactivated the 40 genes predicted to reside between these two markers using RNAi, in the hope that knockdown of the relevant gene would phenocopy gla- 
A

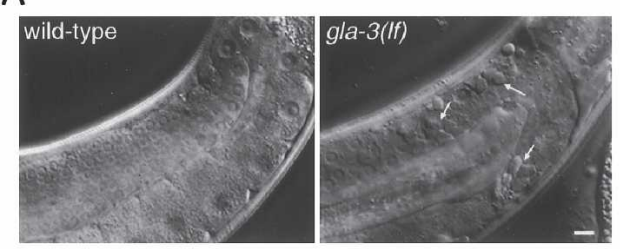

D

B
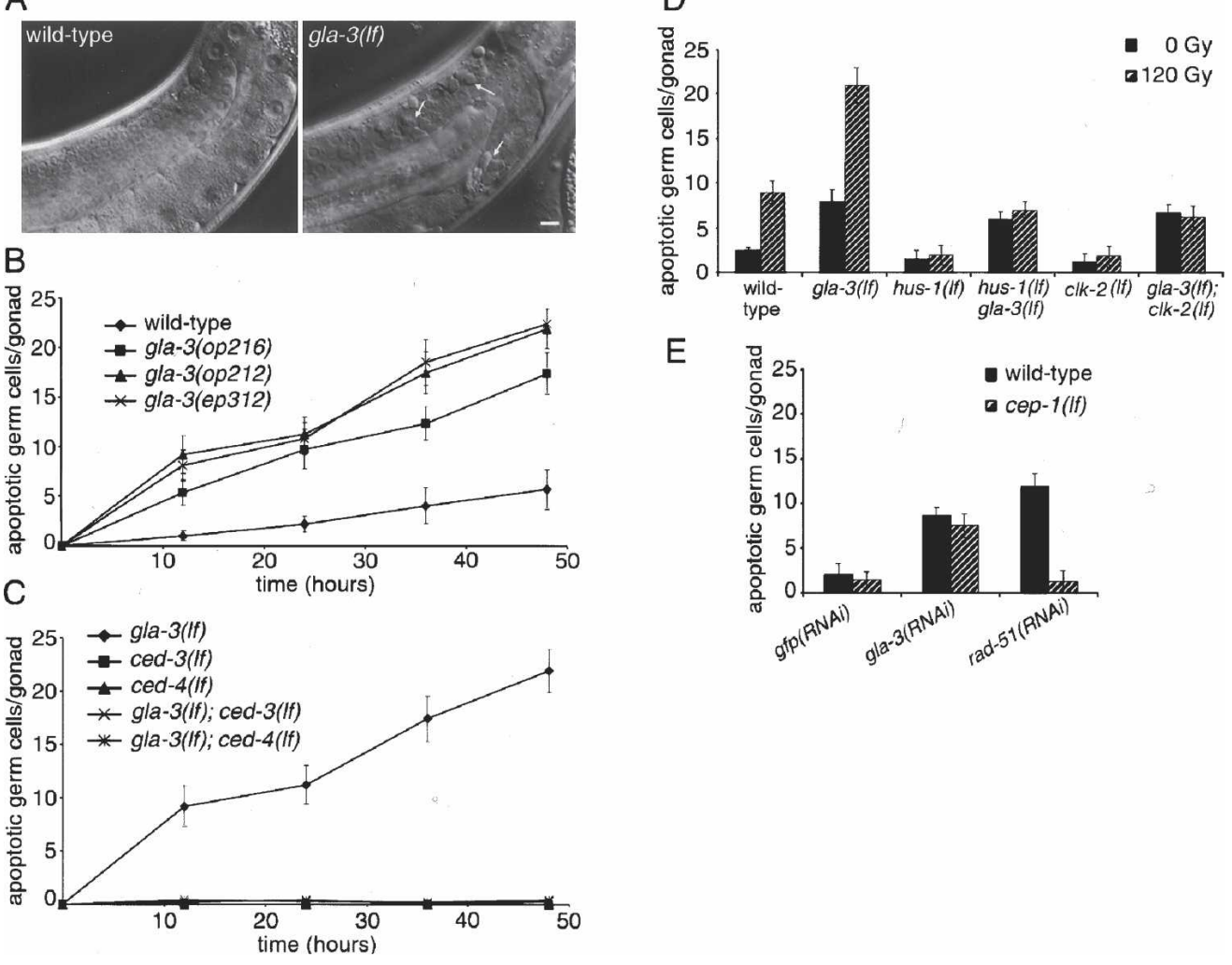

E $\quad$ क wild-type

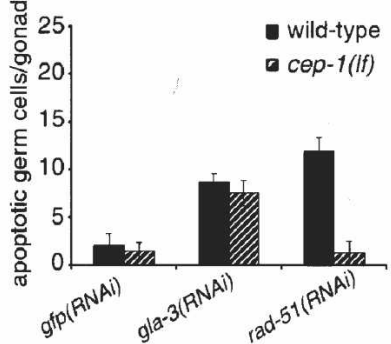

Figure 1. Loss of gla-3 function results in increased germ cell apoptosis. (A) DIC image of wild-type and gla-3(op212) adult hermaphrodite gonads. gla-3(op212) mutants exhibit an increased number of germ cell corpses (arrows). Anterior is to the left and dorsal to the top. Scale bar, $8 \mu \mathrm{m}$. (B) Wild-type and gla-3(lf) mutant worms raised at $25^{\circ} \mathrm{C}$ were synchronized, and germ cell corpses were counted starting at the L4 stage. (C) gla-3(op212)-induced germ cell death is abrogated in ced-4(n1162) and ced-3(n717) mutants. (D) Mutations in DNA damage checkpoint genes do not abrogate gla-3(op212)-induced apoptosis. Synchronized adults (12 h post-L4/adult molt) were irradiated with $120 \mathrm{~Gy}$, and germ cell corpses were counted $24 \mathrm{~h}$ later. The following alleles were used: gla-3(op212), hus-1(op244), and clk-2(mn159ts). (E) gla-3(RNAi)-induced apoptosis is not blocked in cep-1(gk138) mutants. RNAi-mediated knockdown of the RecA homolog rad-51, which results in the accumulation of meiotic intermediates and induces CEP-1-dependent apoptosis (Lettre et al. 2004), was used as a positive control. In panels $B-E$, error bars represent SD from three independent experiments; 20 gonads were scored in each experiment.

3(op212). A single gene, T02E1.3, which encodes two splice variants, gave rise to increased germline apoptosis when inactivated by RNAi. We sequenced T02E1.3 from wild-type, op212, and op216 animals and found a C-to-T transition at position 471 in T02E1.3a (position 574 in T02E1.3b) in op212, resulting in a premature stop codon (Fig. 2A), whereas op216 mutants contain a G-to-A transition in the acceptor splice site of the first intron of T02E1.3a (Fig. 2A). The third allele of gla-3, ep312, was generated in an independent screen for mutants with increased germline apoptosis in a cep-1(lf) mutant background (M. Costa, pers. comm.). ep312 results in a 265bp deletion that removes part of the last two exons of gla-3 and introduces a frameshift at the new junction (Fig. 2A). Based on the strength of its phenotype and the molecular nature of the mutation, we believe op212 to be a null allele of gla-3.

\section{gla-3 encodes a TIS11-like zinc finger domain protein}

gla-3 encodes a protein that contains two $\mathrm{CCCH}$-like zinc-finger domains (Fig. 2B). This domain is found in a subset of zinc-finger family proteins; its consensus sequence corresponds to $\mathrm{C}-\mathrm{X}_{8-10}-\mathrm{C}-\mathrm{X}_{5}-\mathrm{C}-\mathrm{X}_{3}-\mathrm{H}$, where $\mathrm{X}$ refers to any amino acid (Varnum et al. 1989). CCCH zinc fingers were first described in the mouse TIS11 protein, where they are responsible for nucleic-acid binding (Varnum et al. 1989; DuBois et al. 1990; Bai and Tolias 1996). Mammalian TIS11-like zinc-finger-containing proteins have been shown to function either as transcription factors or RNA-binding proteins (Taylor et al. 1996; Worthington et al. 2002). Several C. elegans genes encoding proteins with TIS11-like zinc-finger domains have been described, including pie-1, mex-1, pos-1, mex-5, and mex-6, all of which are involved in early blastomere cellfate determination and germline development (Mello et al. 1996; Guedes and Priess 1997; Tabara et al. 1999; Schubert et al. 2000). In addition, the recently identified TIS11-like proteins OMA-1, OMA-2, and OMA-3 are redundantly required for prophase progression during oocyte maturation (Detwiler et al. 2001; Shimada et al. 2002). GLA-3 is highly similar to these proteins in the $\mathrm{CCCH}$ zinc-finger domain (Fig. 2B), but bears no signifi- 


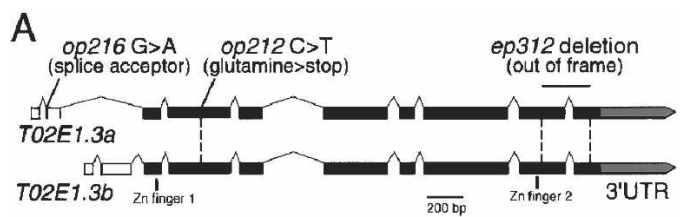

B

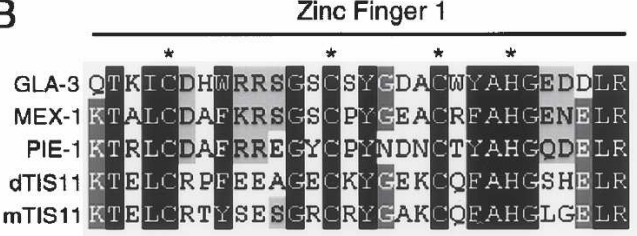

Zinc Finger 2

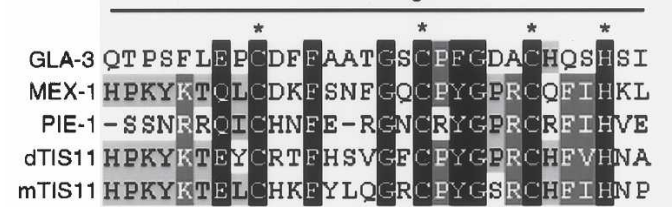

Figure 2. GLA-3 encodes a TIS11-like zinc-finger-containing protein. (A) Schematic representation of gla-3 genomic structure. T02E1.3 is predicted to have two alternatively spliced forms, which differ in their first two exons. Black boxes indicate exons that are common between T02E1.3a and T02E1.3b; the gray arrow indicates the 3 '-untranslated part of the last exon. White boxes represent alternative $5^{\prime}$-exons that are not shared between the two isoforms. Positions of the gla-3(op212), gla3(op216), and gla-3(ep312) lesions are indicated. Size bar, 200 bp. (B) Alignment of the two zinc-finger domains of C. elegans GLA-3, MEX-1, PIE-1, Drosophila melanogaster dTIS11, and mouse mTIS11. Conserved residues are highlighted in black, dark gray, or light gray, depending on whether five out of five, four out of five, or three out of five proteins possess an identical or similar amino acid at this position. Amino acids that coordinate the zinc atom are marked with asterisks.

cant sequence similarity to other characterized proteins outside of the zinc fingers. Unlike most of the characterized CCCH-zinc-finger-containing proteins in C. elegans, the zinc-finger domains in GLA-3 are situated at the two ends of the protein, $\sim 400$ amino acids apart.

\section{gla-3 is expressed in the germline}

We probed a developmental Northern blot from wildtype animals with a probe hybridizing to both gla-3 transcripts. We found gla-3 to be expressed in embryos, L4-stage larvae, and adults (Fig. 3A), with the highest expression being in L4 larvae. The predominant developmental difference between an L4 larva and an adult hermaphrodite is the expansion of the germline. To examine the expression of gla-3 in the adult hermaphrodite germline, we prepared RNA from three temperature-sensitive mutants, defective in various aspects of germline development. At the nonpermissive temperature, glp4(bn2ts) mutants produce a somatic gonad that is largely devoid of germ cells (Beanan and Strome 1992), fem2(e2105ts) mutants make oocytes but no sperm, and fem-3(q20sd) mutants make sperm but no oocytes (Barton et al. 1987). We detected gla-3 expression in all three mutant backgrounds, demonstrating that gla-3 is expressed in both germline and soma (Fig. 3B). Very low levels of mRNA were observed in gla-3(op212) mutants, consistent with the finding that this mutation causes a premature stop, which often results in rapid elimination of the mutant transcript via nonsense-mediated decay (Weischenfeldt et al. 2005).

To investigate the expression pattern of GLA-3 in vivo, we generated strains carrying a GFP reporter driven by the gla-3 promoter. A $1.5-\mathrm{kb}$ genomic fragment upstream of the T02E1.3a ATG was fused to a fragment coding for nuclear localized GFP, and transgenic animals were generated by biolistic transformation (Praitis et al. 2001). GFP expression was observed throughout the germline from the L4 stage onward and in all cells of the early embryo (Fig. 3C), consistent with the gla-3 mRNA pattern in the developmental Northern blot (Fig. 3A). Interestingly, expression was also observed in muscle cells in threefold stage embryos (Fig. 3C, panel c).

To further investigate temporal and spatial gla-3 expression, we raised specific polyclonal antibodies against peptides derived from the unique $\mathrm{N}$ termini of GLA-3A (T02E1.3a) and GLA-3B (T02E1.3b). Western blot analysis of synchronized wild-type worms revealed that both isoforms are expressed at the L4 and adult stages, whereas no expression was detectable from the L1 to L3 stages, nor in gla-3(op212) mutants (Fig. 3D). To examine the expression pattern of GLA-3 in the germline, we investigated the localization of the GLA-3 protein by in situ immunostaining. Consistent with the mRNA expression pattern, GLA-3B was detectable in L4 and adult gonads. The protein was expressed in the mitotic and meiotic zones of the adult germline, and localized to the cytoplasmic compartment, with an enrichment in cortical/submembrane regions (Fig. 3E); a similar pattern of expression was observed with GLA-3A (data not shown). The GLA-3B staining was absent in the gonads of gla3(op212) mutants (Fig. 3E).

\section{Loss of gla-3 results in impaired oocyte development}

gla-3(lf) mutants were morphologically normal, but exhibited a greatly reduced brood size and a low frequency of embryonic lethality (Table 1). Both defects were milder in gla-3(op216) mutants, consistent with the predicted hypomorphic nature of this mutation (which affects only one of the two GLA-3 isoforms). All three mutants showed a further reduction in brood size when raised at high temperature $\left(25^{\circ} \mathrm{C}\right)$ (Table 1$)$. Because this reduction was also apparent in the predicted null mutant gla-3(op212), we surmise that loss of gla-3 function uncovers an intrinsically temperature-sensitive process that is required for oocyte development.

What is the cause of the low brood size in gla-3 mutants? Sperm in gla-3(op212) hermaphrodites and males appeared morphologically normal. We tested the fertility of gla-3(lf) sperm by mating gla-3(lf) males with unc32(e189) hermaphrodites, and found that the mating efficiency of gla-3(lf) males is comparable to that of wildtype animals (Supplemental Table I). Our results indi- 
A

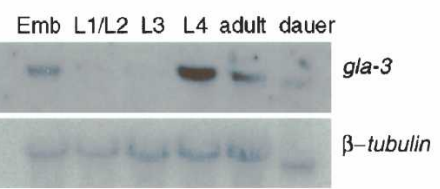

C
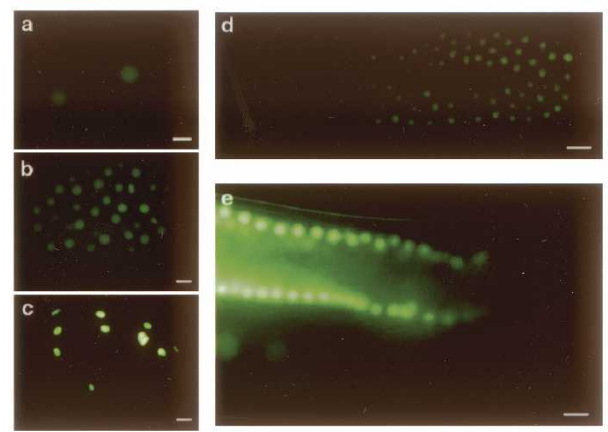

E
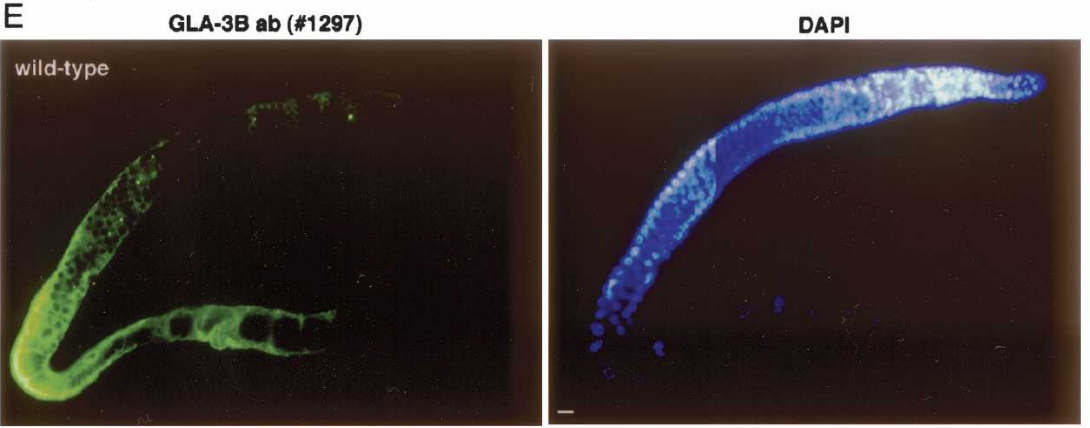

gla-3(t)

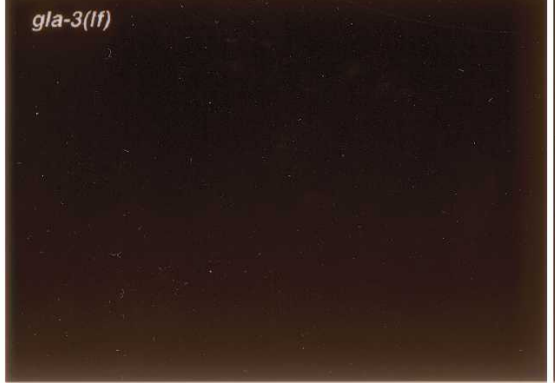

B

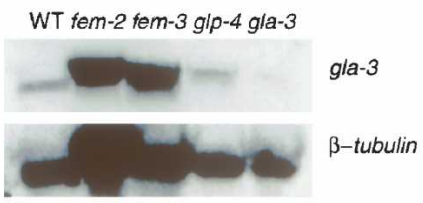

D

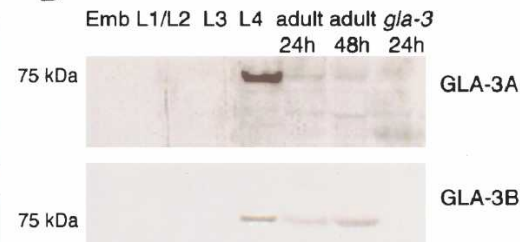

$50 \mathrm{kDa}---\infty-\infty-\alpha$-tubulin

Figure 3. gla-3 expression is developmentally regulated. (A) A Northern blot of total RNA isolated from synchronized wildtype animals was probed with a gla-3 cDNA as described in Materials and Methods. $\beta$-Tubulin was used as a loading control. (B) gla-3 mRNA is present in both germline and soma. $\operatorname{Poly}(\mathrm{A})^{+} \mathrm{RNA}$ was extracted from synchronized wild-type, fem2(e2105ts) (oocytes only), fem-3(q20sd) (sperm only), and glp-4(bn2ts) (no germ cells) adults. $\beta$-Tubulin was used as a loading control. (C) $P_{\text {gla-3a }}:: 2 x N L S:: g f p$ expression in $C$. elegans embryos and germline. Ubiquitous expression was observed in early embryos $(a, b)$ and in the germline at the L4 larval (d) and adult (e) stages. Expression was also observed in muscle cells in threefold embryos $(c)$. (D) Western blot analysis of a developmental time course of wild-type and adult gla-3(op212) worms using isoform-specific anti-GLA-3 antibodies. Total protein was extracted as described in Materials and Methods. Twenty micrograms of total protein was loaded per lane; $\alpha$-tubulin was used as a loading control. (E) Localization of GLA-3B in adult hermaphrodite gonads. Gonads were dissected from wild-type and gla-3(op212) worms $24 \mathrm{~h}$ after the L4/adult molt and were stained with the anti-GLA-3B-specific antibody 1297 and DAPI. Scale bar in $C$ and $E, 8 \mu \mathrm{m}$.

cate that the fertility defects in gla-3(lf) mutants likely stem from an oogenesis defect.

Consistent with the above conclusion, the gonads of gla-3(op212) adult hermaphrodites contained few, abnormally shaped oocytes (Fig. 4A). To examine whether the reduced brood size was the result of excessive apoptosis, we observed gonads of gla-3(op212); ced-3(n717) double mutants. Although apoptosis was completely suppressed in these animals (Figs. 1C, 4A), oogenesis was still impaired, indicating that gla-3 function is necessary for oocyte differentiation rather than germ cell survival per se (Table 1).

To further investigate the oogenesis defect, we stained germ cell nuclei in wild-type and gla-3(lf) worms with 4',6-diamidino-2-phenylindole dihydrochloride (DAPI). In wild-type worms, the transition from mitosis to meiosis occurs at the middle of the distal arm, and pachytene nuclei become visible beyond the transition zone. Oocytes then enter diakinesis in the proximal arm of the gonad, where highly condensed chromosomes can be seen (Fig. 4B). The distal gonads of gla-3(op212) mutants were indistinguishable from those of wild-type animals when observed under Nomarski optics or by DAPI staining (Fig. 4A,B). However, only few oocytes developed to the diakinesis stage in the proximal gonads of gla3(op212) adults; detailed analysis revealed that this region was filled mostly with pachytene-stage nuclei (Fig.

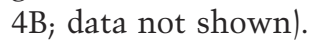

Inhibition of apoptosis did not significantly improve the differentiation defects that are caused by loss of gla-3 function: DAPI staining of gla-3(op212); ced-3(n717) double mutants revealed an unusually large number of pachytene-stage nuclei in the proximal arm (Fig. 4; data not shown). The double mutant did contain more diaki- 
Kritikou et al.

Table 1. gla-3(lf) mutants have a reduced brood size and suffer from embryonic lethality

\begin{tabular}{lcccc}
\hline & \multicolumn{2}{c}{ Brood size } & \multicolumn{2}{c}{ Survival $(\%)$} \\
\cline { 2 - 3 } Genotype & $20^{\circ} \mathrm{C}(n=17)$ & $25^{\circ} \mathrm{C}(n=15)$ & $20^{\circ} \mathrm{C}(n=20)$ & $25^{\circ} \mathrm{C}(n=15)$ \\
\hline Wild type & $259 \pm 18$ & $264 \pm 23$ & $98 \pm 2$ & $99 \pm 5$ \\
ced-3(n717) & $263 \pm 22$ & $280 \pm 18$ & $98 \pm 5$ & $96 \pm 6$ \\
gla-3(op212) & $20 \pm 3$ & $5.7 \pm 2.1$ & $81 \pm 7$ & $75 \pm 3$ \\
gla-3(ep312) & $12 \pm 5$ & $4.8 \pm 1.4$ & $95 \pm 8$ & $90 \pm 4$ \\
gla-3(op216) & $180 \pm 25$ & $112 \pm 45$ & $85 \pm 3$ & $81 \pm 5$ \\
gla-3(op212); ced-3(n717) & $14 \pm 3$ & $11 \pm 2$ & $75 \pm 10$ & $79 \pm 6$ \\
gla-3(ep312); ced-3(n717) & $13 \pm 5$ & $10 \pm 3$ & $90 \pm 8$ & $91 \pm 5$ \\
gla-3(op216); ced-3(n717) & $100 \pm 20$ & $53 \pm 31$ &
\end{tabular}

Brood size and survival rate were scored as previously described (Hofmann et al. 2002). The data shown are the average \pm SD. (n) Number of broods analyzed.

nesis stage oocytes than the gla-3 single mutant; however, these diakinetic oocytes were misshapen and abnormally small. These results indicate that inhibition of apoptosis allows more female germ cells to enter diakinesis in gla-3 mutants, but does not rescue the gla-3(lf) differentiation defect(s).

\section{Biochemical interaction of GLA-3 and MPK-1 in vitro and in vivo}

To identify molecular links between GLA-3 and other proteins involved in oogenesis, we searched the worm interactome (WI5, http://vidal.dfci.harvard.edu/interactomedb) for interaction partners of GLA-3, and found a yeast two-hybrid $(\mathrm{Y} 2 \mathrm{H})$ interaction between GLA-3 and the two isoforms of MPK-1. Using these two MPK-1 isoforms as baits, both GLA-3 isoforms were found as the predominant preys (over $50 \%$ of hits) in the AD-cDNA and AD-ORFeome prey libraries (Li et al. 2004; data not shown). MPK-1 is the C. elegans homolog of the mammalian ERK1/2 serine/threonine kinases. MPK-1 is required for germline development: In mpk-1(lf) mutants, germ cells fail to exit the pachytene stage of meiosis I, resulting in sterility and decreased germ cell apoptosis (Church et al. 1995; Lackner and Kim 1998; Gumienny et al. 1999).
To verify the Y2H result, we performed glutathione-Stransferase (GST) affinity purification experiments in $293 \mathrm{~T}$ cells transfected with MPK-1 fused to GST and myc-tagged GLA-3. Both isoforms of GLA-3 interacted with both isoforms of MPK-1 (Fig. 5A). To validate the in vitro evidence and show that MPK-1 interacts with GLA-3 in vivo, we immunoprecipitated GLA-3 from $C$. elegans extracts and tested for MPK-1 binding. As illustrated in Figure 5B, MPK-1 could be immunoprecipitated with both isoforms of GLA-3. Our findings show a direct interaction between GLA-3 and MPK-1 in vitro and in vivo, and provide a possible explanation for the germline defects observed in gla-3(lf) mutants.

To define the GLA-3 binding interface on MPK-1, we used the reverse $\mathrm{Y} 2 \mathrm{H}$ system. We screened a PCR-mutagenized library of $m p k-1$ and selected for mutants that could no longer interact with GLA-3. From this set of mutants, we analyzed further those with a single, nonsilent nucleotide change. In total, we identified 20 unique amino acid substitutions that represent 17 different amino acid positions on MPK-1 (Table 2). The majority (nine out of 17) of the amino acid changes that lead to a disruption of the interaction between MPK-1 and GLA-3 clustered to a small region on the surface of MPK-1 (Fig. 6). The region defined by these residues is structurally conserved between MPK-1 and ERK2 and
Figure 4. Loss of gla-3 function results in meiotic arrest during oogenesis. (A) Wildtype, gla-3(op212), and gla-3(op212); ced3(n717) adult hermaphrodite animals were examined using DIC microscopy. In wildtype worms, oocytes enter diakinesis in the proximal arm of the gonad (arrow). Arrowheads indicate apoptotic corpses in gla-3(lf) animals. (B) Images of DAPIstained gonads from wild-type, gla3(op212), and gla-3(op212); ced-3(n717) adult hermaphrodites. Pachytene nuclei are evident around the bend of the gonad arm. The proximal arm of gla-3(op212) mutants contained an excess of pachytene nuclei compared to wild-type worms. The asterisk indicates the first diakinetic nucleus in the proximal arm of gla-3(lf); ced-3(lf). Scale bar, $8 \mu \mathrm{m}$.
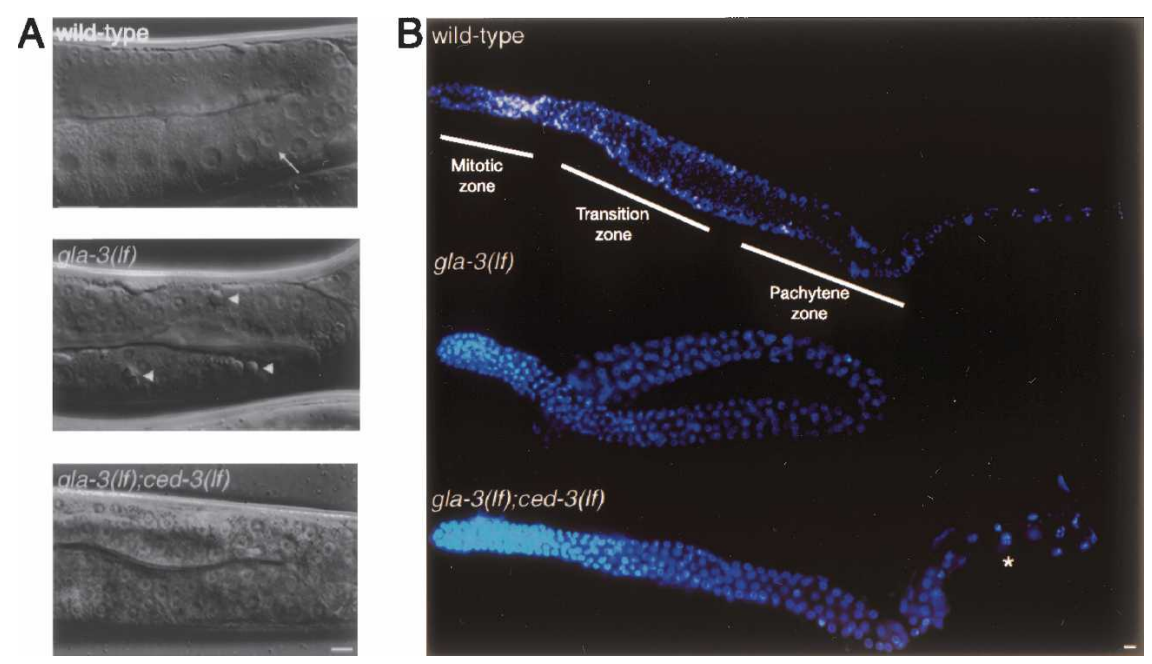


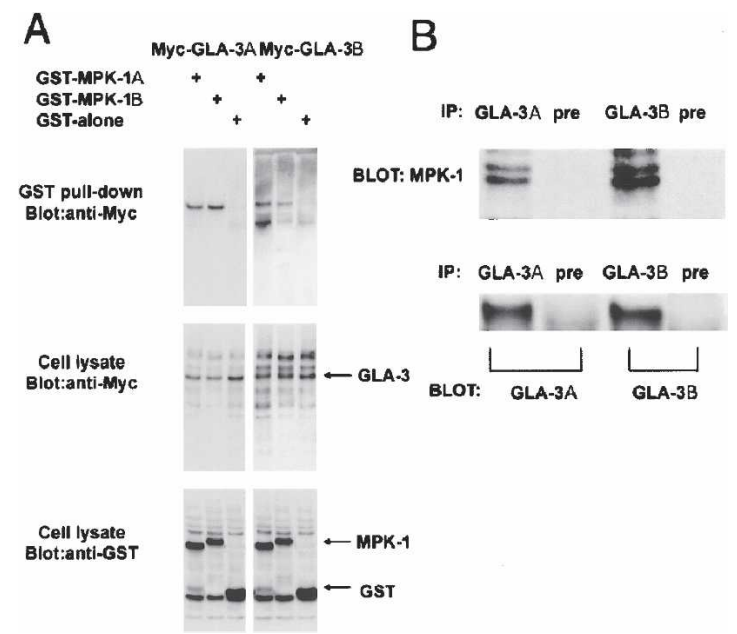

Figure 5. MPK-1 and GLA-3 interact in vitro and in vivo. $(A)$ Myc-GLA-3 and GST-MPK-1 interact in 293T cells. 293T cells were transfected with plasmids expressing Myc-tagged GLA-3A or GLA-3B isoforms and GST-MPK-1A or GST-MPK-1B. GSTMPK-1A or GST-MPK-1B was affinity-purified using glutathione beads, and the presence of Myc-GLA-3A or Myc-GLA-3B was detected by immunoblotting using an anti-Myc antibody. (B) MPK-1 interacts with GLA-3 in C. elegans. Total protein was extracted from synchronized adult hermaphrodites, and immunoprecipitation was performed using anti-GLA-3A or GLA3B antibodies. Pre-immune serum was used as a control. Immunoprecipitated samples were probed with an antibody against mammalian ERK1/2, which recognizes C. elegans MPK-1 (top panel) (Lackner and Kim 1998) and with antibodies against GLA-3A or GLA-3B (bottom panel).

has been shown to function as a docking site for both regulators and targets of MAPK, such as the phosphatase MKP3 and the kinase ELK1 (Table 2; Zhang et al. 2003). In addition, GLA-3 contains potential MPK-1 binding sites, as defined by a cluster of positive amino acids surrounded by hydrophobic amino acids (Tanoue and Nishida 2002). GLA-3a has one site at position 66-74 (LRKVVRIDR), whereas GLA-3b has two such sites, at positions 66-74 (LRKTKI) and 99-107 (LRKVVRIDE). Taken together, our biochemical and molecular data are consistent with a role of GLA-3 as a regulator or a target of MPK-1 in the C. elegans germline.

gla-3 genetically interacts with components of the MAPK signaling pathway

Do ras/MAPK pathway genes regulate the apoptotic program? Strong loss-of-function mutations in the mpk-1 pathway cause a sterile phenotype due to developmental arrest at the pachytene stage of meiosis /Church et al. 1995). We and others have previously shown that pachytene-arrested cells do not undergo apoptosis (Gumienny et al. 1999; Navarro et al. 2001). To address the role of mpk-1 in gla-3(lf)-induced cell death, we performed genetic epistasis analysis between $g l a-3$ and genes that are known to be involved in the Ras/MAPK pathway. Using the putative null mutation mpk-1(ga117) (Lackner and
Kim 1998), we found that gla-3(op212)-induced apoptosis was completely abrogated in gla-3(op212); mpk1(ga117) double mutants (Fig. 7A). Interestingly, although apoptosis is suppressed, gla-3 does not rescue the sterile phenotype of mpk-1(ga117) (Supplemental Table II). These data demonstrate that $m p k-1$ is epistatic to gla-3, and that its activation is required for gla-3(lf)-induced germ cell death. We then compared the levels of germ cell death in mpk-1(oz140) ced-9(n2812) adult hermaphrodites with those in gla-3(RNAi); mpk-1(oz140) ced-9(n2812) animals. ced-9(lf)-induced and gla3(RNAi)-induced germ cell apoptosis were both completely suppressed in the absence of functional $m p k-1$ (Gumienny et al. 1999; Supplemental Table III).

We also analyzed the effect on germline apoptosis of mutants that cause overactivation of MPK-1. We observed no increase in germ cell apoptosis in the ras gainof-function mutant let-60(ga89). Similarly, gla-3(op212); let-60(ga89) double mutants had levels of apoptosis that were comparable to gla-3(op212) alone (Fig. 7B). In contrast, inactivation of LIP-1, an MPK-1 phosphatase that functions as a negative regulator of MAPK signaling (Hajnal and Berset 2002), resulted in increased germline apoptosis, with levels comparable to gla-3 mutants (Fig. 7B). Similarly to gla-3 mutants, lip-1(zh15)-induced germline apoptosis depended on ced-3 but was cep-1independent (Supplemental Fig. 3), indicating that these two genes might function in the same genetic pathway. Indeed, gla-3(op212); lip-1(zh15) double mutants showed only a slight increase in germline apoptosis over the single mutants (Fig. 7B).

lip-1(zh15) mutants display accelerated oocyte devel-

Table 2. Point mutations in MPK-1 that disrupt interaction with GLA-3

\begin{tabular}{lrlll}
\hline Position $^{\mathrm{a}}$ & Hits & Wild type & Mutant & DS $^{\mathrm{c}}$ \\
\hline 130 & 1 & Ser (TCT) & Pro (CCT) & No \\
131 & 1 & Ser (TCT) & Phe (TTT) & No \\
253 & 14 & Asn (AAT) & Asp (GAT) & Yes \\
391 & 1 & Tyr $^{\mathrm{b}}$ (TAC) & His (CAC) & Yes \\
391 & 1 & Tyr $^{\mathrm{b}}$ (TAC) & Asp (GAC) & Yes \\
398 & 3 & Leu (CTC) & Pro (CCC) & Yes \\
410 & 1 & Leu (CTC) & Pro (CCC) & Yes \\
413 & 1 & Argb (CGT) & His (CAT) & Yes \\
433 & 1 & Ser (TCT) & Pro (CCT) & No \\
473 & 1 & Leu (TTG) & Ser (TCG) & No \\
479 & 1 & Leu (CTC) & Pro (CCC) & Yes \\
490 & 1 & Cys (TGT) & Ser (AGT) & Yes \\
491 & 1 & Cys (TGT) & Tyr (TAT) & Yes \\
497 & 1 & Leu (CTC) & Pro (CCC) & Yes \\
653 & 1 & Gly (GGA) & Glu (GAA) & No \\
655 & 1 & Cys (TGT) & Arg (CGT) & No \\
692 & 1 & Phe (TTC) & Ser (TCT) & No \\
719 & 1 & Leu (CTC) & Pro (CCC) & No \\
896 & 1 & Phe (TTC) & Ser (TCC) & No \\
955 & 1 & Tyrb (TAC) & His (CAC) & Yes \\
\hline
\end{tabular}

aposition indicates nucleotide position based on MPK-1 isoform a. ${ }^{\mathrm{b}}$ Residues necessary for docking that are identical in MPK- 1 and ERK2.

${ }^{\mathrm{c}}$ Amino acids that are part of the predicted MPK-1 docking site. 

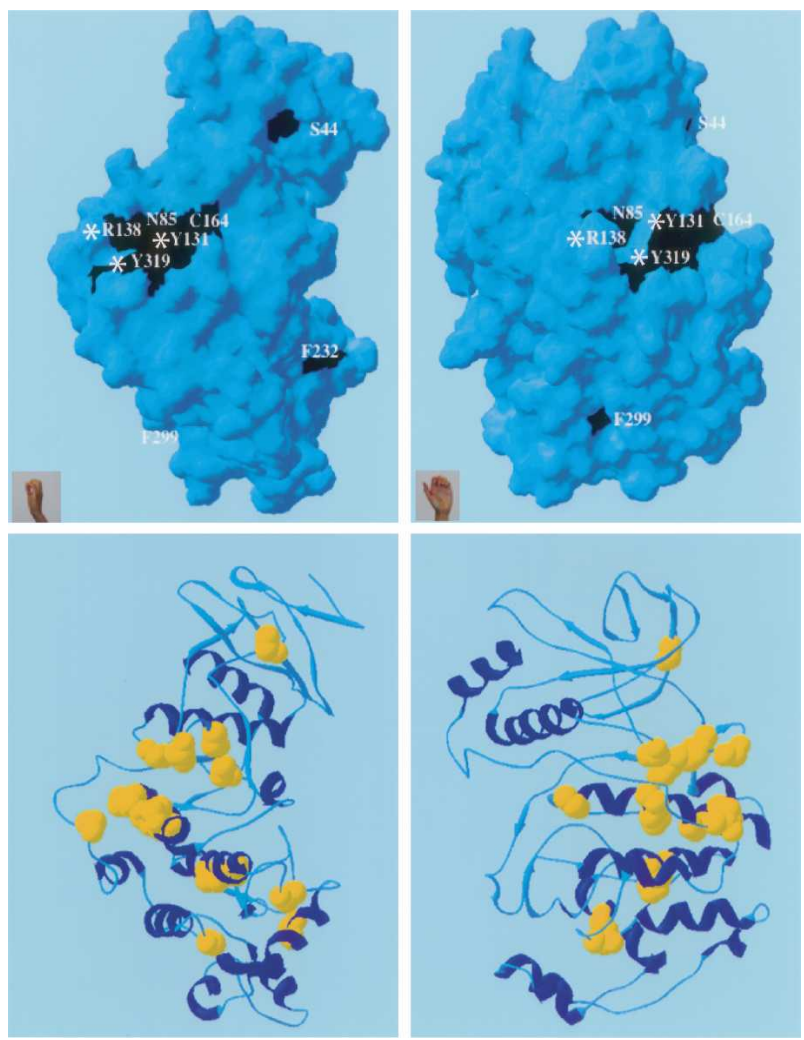

Figure 6. Identification of MPK-1 residues required for the interaction with GLA-3. The reverse two-hybrid system was used to identify residues that are essential for the MPK-1/GLA-3 interaction. Residues, which when altered result in disruption of the interaction, are shown in black in the space-filling model (top panels) and in yellow in the ribbon model (bottom panels) of MPK-1. Residues with visible surface exposure are noted on the structure, and those that are identical between MPK-1 and its mammalian homolog ERK2 are noted with an asterisk. Left and right panels represent a $90^{\circ}$ rotation of the structure on its axis.

opment due to defective $\mathrm{G}_{2} / \mathrm{M}$-phase arrest (Hajnal and Berset 2002). We scored the number of diakinetic oocytes in the gonads of single and gla-3(op212); lip-1(zh15) double mutants and found that the double mutants exhibited decreased numbers of diakinetic oocytes, a phenotype similar to gla-3(op212) single mutants (Supplemental Table II). These observations suggest that gla-3 is epistatic to lip-1 for germ cell differentiation.

\section{gla-3 mutations do not result in MPK-1 overactivation}

To test whether loss of gla-3 function altered the pattern of MPK-1 activation in the germline, we stained dissected gonads with a monoclonal antibody that specifically recognizes the diphosphorylated, activated form of MPK-1 (DP-MPK-1). As previously reported (Miller et al. 2001; Page et al. 2001), MPK-1 was activated in pachytene-stage cells and also, in response to the sperm signal, in the first and sometimes second oocyte proximal to the spermatheca (Supplemental Fig. 4A). The pattern of
MPK-1 activation in gla-3(op212) mutants was similar to that of wild-type animals, although the DP-MPK-1 staining in the oocytes proximal to the spermatheca was occasionally weaker (Supplemental Fig. 4A), possibly reflecting the abnormal nature of oocytes in gla-3 mutants.

To further test the hypothesis that GLA-3 might regulate the levels of activated MPK-1, we used an in vitro MPK-1 kinase assay (Alessi et al. 1995; Berset et al. 2001). Using myelin basic protein (MBP) as an MAPK substrate, we found that both lip-1(zh15) and let60(n1046gf) animals had higher levels of MAPK enzymatic activity when compared with the wild type (Supplemental Fig. 4B). In contrast, the level of MAPK activity in gla-3(op212) and gla-3(op216) mutants was similar to the wild type (Supplemental Fig. 4B). Taken together, these data indicate that inactivation of GLA-3 does not directly affect the activation of MPK-1 protein itself.

\section{GLA-3 is a negative regulator of the MAPK signaling} pathway in somatic tissues

During C. elegans development, LET-60/Ras and MPK1/MAPK transmit the LIN-3/EGF inductive signal in
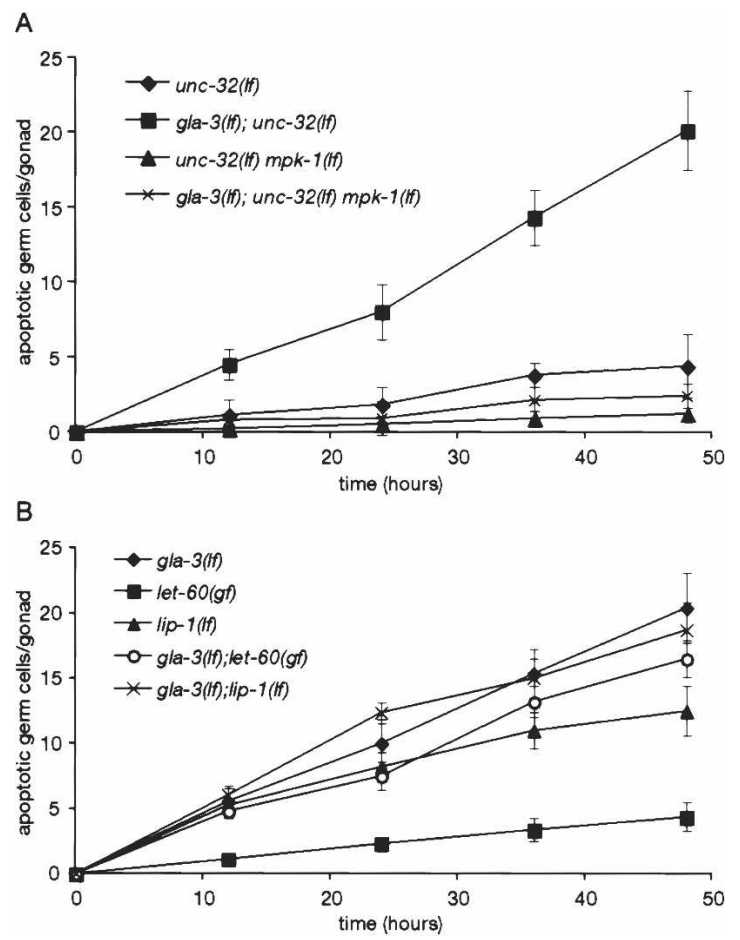

Figure 7. Genetic interactions between gla-3 and genes involved in Ras/MAPK signaling. (A) unc-32(e189), gla-3(op212); unc-32(e189), unc-32(e189) mpk-1(ga117), and gla-3(op212); unc-32(e189) mpk-1(ga117) animals were synchronized, and germ cell corpses were counted over $48 \mathrm{~h}$ starting at the L4 stage. (B) Synchronized gla-3(op212), let-60(ga89), lip-1(zh15), gla-3(op212); let-60(ga89), and gla-3(op212); lip-1(zh15) animals were raised at $25^{\circ} \mathrm{C}$, and germline corpses were counted starting at the L4 stage. Error bars represent SD from three independent experiments; 20 gonads were scored in each experiment. 
Table 3. gla-3 is a negative regulator of vulva induction

\begin{tabular}{|c|c|c|c|}
\hline Genotype & $\begin{array}{c}\% \\
\text { Muv }\end{array}$ & $\begin{array}{c}\text { Average } \\
\mathrm{P}(3-8) \cdot \mathrm{p} \\
\text { vulval fates }\end{array}$ & $\begin{array}{l}\text { Number } \\
\text { of animals }\end{array}$ \\
\hline Wild type & 0 & 3.0 & 75 \\
\hline gla-3(op212) & 0 & 3.0 & 69 \\
\hline gla-3(op216) & 0 & 3.0 & 74 \\
\hline let-60(n1046sd) & 80 & 4.0 & 54 \\
\hline let-60(ga89) & 42 & 3.5 & 64 \\
\hline lip-1(zh15) & 0 & 3.0 & 60 \\
\hline unc-32(e189) & 0 & 3.0 & 45 \\
\hline unc-32(e189) mpk-1(ga117) & 0 & 0 & 54 \\
\hline gla-3(op212); let-60(n1046) & 97 & 4.9 & 48 \\
\hline gla-3(op216); let-60(n1046) & 91 & 5.1 & 52 \\
\hline gla-3(op212); let-60(ga89) & 66 & 4.5 & 68 \\
\hline gla-3(op216); let-60(ga89) & 63 & 4.4 & 84 \\
\hline gla-3(op212); lip-1(zh15) & 4.5 & 3.3 & 66 \\
\hline $\begin{array}{l}\text { gla-3(op216); lip-1(zh15) } \\
\text { gla-3(op212); unc32(e189) }\end{array}$ & 3.8 & 3.1 & 71 \\
\hline $\begin{array}{l}\text { mpk-1(ga117) } \\
\text { gla-3(op216); unc-32(e189) }\end{array}$ & 0 & 0 & 65 \\
\hline mpk-1(ga117) & 0 & 0 & 58 \\
\hline
\end{tabular}

vulval precursor cells (VPCs), resulting in vulva formation (Moghal and Sternberg 2003). Loss-of-function mutations in genes that mediate the inductive signaling pathway can lead to a vulvaless (Vul) phenotype due to insufficient induction of VPCs. Conversely, mutations that enhance the inductive signal result in a multivulva (Muv) phenotype, due to hyperinduction of VPCs.

To test the possibility that GLA-3 might also influence MAPK signaling during vulval development, we examined double mutants between gla-3(lf) and various mutations that affect vulval induction. In gla-3(lf) single mutants, vulval development appeared normal (Table 3). However, loss of gla-3 function enhanced the multivulva phenotype observed in two different let-60(gf) mutants (Table 3), suggesting that GLA-3 might function as an inhibitor of MAPK signaling in VPCs. Similarly, vulval development is normal in lip-1(lf) mutants (Berset et al.
2001), whereas double gla-3(op212); lip-1(zh15) mutants displayed a weak synthetic Muv phenotype (Table 3). In contrast, the gla-3 alleles could not suppress the mpk1(ga117) vulvaless phenotype. Taken together, these results indicate that GLA-3 might function as an inhibitor of MAPK signaling during vulval development.

Activation of the Ras/MAPK pathway has also been shown to promote protein degradation in C. elegans muscles, leading to a progressive loss of motility (Szewczyk et al. 2002). We therefore investigated whether gla-3 might influence muscle-protein degradation through its interaction with the Ras/MAPK pathway. Whereas gla-3 mutants raised at $15^{\circ} \mathrm{C}$ showed nearly wild-type movement, animals shifted to $25^{\circ} \mathrm{C}$ at the $\mathrm{L} 4$ stage suffered a progressive loss of motility, similar to the defect observed in let-60(ga89) mutants (Table 4). The locomotion defect was not significantly enhanced in the gla-3(lf); let-60(lf) double mutants, implying that the two genes might affect the same molecular process.

We then monitored protein degradation using an unc54:: LacZ transgene that is expressed specifically in body wall and vulval muscles (Zdinak et al. 1997; Szewczyk et al. 2000). This fusion protein is completely stable in well-fed wild-type worms; therefore, any subsequent decline in LacZ activity is the result of protein degradation. Well-fed gla-3(op212) mutants exhibited wild-type levels of LacZ activity when grown at $15^{\circ} \mathrm{C}$. In contrast, mutants shifted to $25^{\circ} \mathrm{C}$ at the L4 stage showed a timedependent loss of reporter activity similar to the loss observed in mutants with an overactivated Ras/MAPK pathway (Fig. 8). Taken together, these observations indicate that GLA-3 might negatively regulate Ras/MAPKdependent protein degradation in muscle cells.

\section{Discussion}

Although much is known about the core apoptotic machinery and the signals that regulate apoptosis during $C$. elegans development (for review, see Lettre and Hengartner 2006), the factors that regulate germline apoptosis are largely unknown. In an attempt to isolate such regu-

Table 4. gla-3(1f) causes time-dependent loss of mobility

\begin{tabular}{|c|c|c|c|c|c|}
\hline \multirow[b]{2}{*}{ Genotype } & \multirow[b]{2}{*}{ Temp $\left({ }^{\circ} \mathrm{C}\right)$} & \multicolumn{4}{|c|}{ Movement rate (waves/min) } \\
\hline & & 0 & 24 & 48 & 72 \\
\hline Wild type & 15 & $98 \pm 14$ & $96 \pm 12$ & $108 \pm 8$ & $103 \pm 5$ \\
\hline \multirow{3}{*}{ g1a-3(op212) } & $15 \rightarrow 25$ & & $99 \pm 8$ & $110 \pm 17$ & $102 \pm 9$ \\
\hline & 15 & $100 \pm 8$ & $97 \pm 15$ & $93 \pm 19$ & $109 \pm 8$ \\
\hline & $15 \rightarrow 25$ & & $78 \pm 16$ & $61 \pm 10$ & $57 \pm 12$ \\
\hline \multirow[t]{2}{*}{ g1a-3(op216) } & 15 & $109 \pm 11$ & $103 \pm 8$ & $97 \pm 5$ & $102 \pm 8$ \\
\hline & $15 \rightarrow 25$ & & $82 \pm 7$ & $63 \pm 11$ & $60 \pm 7$ \\
\hline \multirow[t]{2}{*}{ let-60(ga89) } & 15 & $100 \pm 14$ & $95 \pm 15$ & $98 \pm 2$ & $101 \pm 14$ \\
\hline & $15 \rightarrow 25$ & & $83 \pm 9$ & $56 \pm 15$ & $54 \pm 10$ \\
\hline \multirow[t]{2}{*}{ gla-3(op212); let-60(ga89) } & 15 & $90 \pm 9$ & $99 \pm 11$ & $97 \pm 9$ & $89 \pm 12$ \\
\hline & $15 \rightarrow 25$ & & $71 \pm 5$ & $54 \pm 7$ & $48 \pm 11$ \\
\hline \multirow{2}{*}{ gla-3(op216); let-60(ga89) } & 15 & $96 \pm 13$ & $100 \pm 7$ & $106 \pm 4$ & $94 \pm 6$ \\
\hline & $15 \rightarrow 25$ & & $76 \pm 13$ & $62 \pm 7$ & $61 \pm 4$ \\
\hline
\end{tabular}

Data shown are average \pm SD from two independent experiments; 15 animals were scored per experiment. 
Kritikou et al.

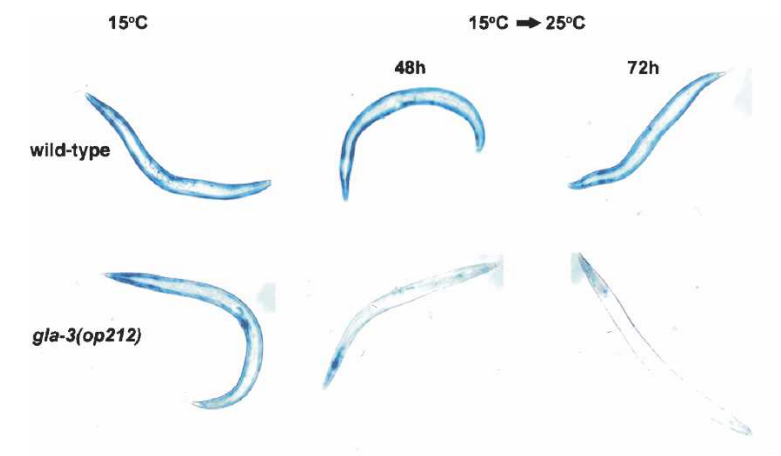

Figure 8. gla-3(lf) promotes proteolysis in body wall muscles. Animals were raised at $15^{\circ} \mathrm{C}$ until the young adult stage (left panel), and then shifted to $25^{\circ} \mathrm{C}$ for 48 or $72 \mathrm{~h}$ (right panels). $\beta$-Galactosidase activity (blue) was measured as described in Materials and Methods. Scale bar, $0.2 \mathrm{~mm}$.

lators, we performed a genetic screen and identified gla3 . We found that gla-3 is important for oocyte differentiation and germ cell survival and encodes a TIS11-like zinc-finger-containing protein. We showed that GLA-3 genetically and physically interacts with the C. elegans MAPK MPK-1 and provided evidence to support the notion that GLA-3 might function as a negative regulator of the MAPK pathway in the developing vulva and in muscle cells.

\section{Regulation of germline apoptosis by GLA-3 and other RNA-binding proteins}

We showed that gla-3 has two isoforms, which differ in their two $5^{\prime}$-most exons. Are both isoforms involved in germline apoptosis and oocyte development? Both GLA3A and GLA-3B are expressed in the germline, and both isoforms interact with MPK-1. Furthermore, the op216 mutation, which is predicted to affect only GLA-3A, shows weaker defects than the predicted null alleles. Based on these results, we suggest that both isoforms might participate in germline development and apoptosis. However, further work is required to confirm this hypothesis, and to determine whether the isoform-specific N-terminal domains perform distinct functions.

How could GLA-3 regulate germ cell apoptosis? The TIS11-like proteins that have previously been characterized in C. elegans, including PIE-1, POS-1 MEX-1, MEX5 , and MEX-6, function in a hierarchical regulatory cascade that controls cell fate during early embryonic development (Schneider and Bowerman 2003). In addition, the combined loss of the TIS11-like proteins, OMA-1 and OMA-2, results in impaired oocyte maturation and female infertility (Detwiler et al. 2001). The mammalian TIS11 zinc-finger-containing protein, tristetraprolin (TTP), is an RNA-binding protein that interacts with 3'untranslated AU-rich elements of mRNAs and negatively regulates their expression (Lai et al. 1999; Carballo et al. 2000; Cao et al. 2003). Based on the homology between the C. elegans and the mammalian TIS11-like proteins, it is possible that PIE-1, POS-1, MEX-1, MEX-5, MEX-6, and GLA-3 exert their functions by acting as mRNA-binding proteins (Barabino et al. 1997; Lai et al. 2000; Worthington et al. 2002).

Inactivation of several RNA-binding proteins, including DAZ-1, CGH-1, CPB-3, and CAR-1, has previously been shown to trigger apoptosis in the C. elegans germline (Karashima et al. 2000; Navarro et al. 2001; Lettre et al. 2004; Boag et al. 2005). Similarly to gla-3, daz-1 mutations result in severe oogenic defects due to arrest at the pachytene stage of meiosis I. It is possible that these genes coordinately regulate the synthesis of pro-apoptotic or survival proteins during germline development. Indeed, CGH-1 and CAR-1 interact physically and are components of an evolutionarily conserved RNP complex (Boag et al. 2005). Alternatively, these proteins might function more broadly to promote germline differentiation, and the developmental defects that result from their loss could be the cause of the apoptotic phenotypes observed. Identification of the target mRNAs regulated by these various proteins is required to shed light on this issue.

\section{GLA-3 is a novel component of the MAPK signaling cascade}

In addition to its potential function as an RNA-binding protein, GLA-3 also interacts physically with the C. elegans MAPK MPK-1 in vitro and in vivo, indicating that GLA-3 might have a role in MPK-1 signaling. In the hermaphrodite germline, activation of the LET-60/MEK-2/ MPK-1 signaling pathway is essential for pachytene progression and subsequent entry into diplotene/diakinesis (Church et al. 1995; Lackner and Kim 1998; Miller et al. 2001; Page et al. 2001). Although MPK-1 activation during pachytene progression appears to be transient and tightly regulated (Hajnal and Berset 2002), the nature of the signal that controls its activation and the downstream effectors of MPK-1 remain poorly characterized. Based on its mutant phenotype and its physical interaction with MPK-1, GLA-3 might be directly involved in this process.

Because our expression studies revealed that GLA-3 expression is not restricted to the germline, we also studied the potential role of GLA-3 in MPK-1 signaling in the soma. We found that the absence of gla-3 resulted in phenotypes consistent with an inappropriate activation of mpk-1 signaling in both vulval development and protein degradation in body wall muscles. These biochemical and genetic analyses support the idea that GLA-3 functions as a negative component of the Ras/MAPK pathway. However, we were so far unable to detect any change in MPK-1 activity in gla-3 mutants or worm extracts (Supplemental Fig. 4), suggesting that GLA-3 does not directly regulate MPK-1 activation but, rather, might restrict its access to or interaction with substrates.

Could GLA-3 be a substrate rather than-or in addition to-a regulator of MPK-1? Neither our genetic nor our biochemical data allow us to definitively exclude this possibility. We did not observe any differences in the 
GLA-3 protein levels in different mutants of the MAPK pathway by Western blot analysis and in situ immunostaining (Supplemental Fig. 5; data not shown), which indicated that MPK-1 does not directly regulate GLA-3 levels. However, it is possible that MPK-1 phosphorylates GLA-3 and this modification is important for GLA-3 function. Indeed, several kinases, including MAPKMAP kinase 2 (MK2), ERK2, p38, and JNK, can phosphorylate the mammalian TIS11 protein tristetraprolin in response to growth factor or cytokine treatment (Taylor et al. 1995; Mahtani et al. 2001; Chrestensen et al. 2004). Although GLA-3 has several potential MAPK phosphorylation sites, we were unable to determine whether GLA-3 is phosphorylated by MPK-1 in vivo using the antibody that we raised (Supplemental Material 5).

What is the function of GLA-3 in the MAPK signaling pathway?

In Schizosaccharomyces pombe, MAPK signaling activity can be regulated through a negative feedback loop that involves Rnc1, a KH-domain-containing RNA-binding protein that stabilizes the Pmp1 phosphatase mRNA (Sugiura et al. 2003). Based on the ability of GLA-3 to bind to MPK-1 and its predicted RNA-binding domains, a similar model could be envisaged in which GLA-3 is activated through interaction with MPK-1, and in response stabilizes mRNAs encoding negative regulators of MPK-1 such as LIP-1.

\section{Materials and methods}

Strains, alleles, and genetic analysis

Maintenance and genetic manipulation of C. elegans were carried out as described (Brenner 1974). The following mutations and transgenes were used: LG I: hus-1(op244), gla-3(op212), gla-3(op216), gla-3(ep312), cep-1(gk138), glp-4(bn2ts); LG II: lip-1(zh15); LG III: fem-2(e2105ts), mpk-1(ga117), mpk1(oz140), ced-4(n1162), clk-2(mn159ts), unc-32(e189), ced-

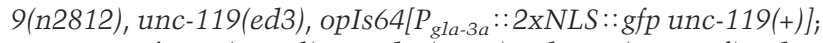
LG IV: fem-3(q20sd), ced-3(n717), let-60(n1046gf), let60(ga89gf), opIs110[P $P_{\text {lim- }-7}:: y f p:: a c t-5$ unc-119(+)]; LG V: ccIs55[unc-54::1acZ; sup-7(st5)], gaIs36[HS-mpk-1(+) EF1-Dmek(+) unc-30(+)]. All mutations are described in WormBase (http://www.wormbase.org). The Bristol strain N2 was used as the wild-type strain. Strains were maintained at $20^{\circ} \mathrm{C}$ unless stated otherwise. Unless specified otherwise, all phenotypic characterizations were performed on hermaphrodites $24 \mathrm{~h}$ postL4/adult molt. Movement rates were measured as described (Bolanowski et al. 1981).

\section{Isolation of gla-3 alleles}

gla-3(lf) alleles were isolated in an $F_{2}$ screen for mutants with increased germ cell apoptosis. Wild-type animals were mutagenized with ethylmethane sulfonate using a standard protocol (Brenner 1974), and adult $F_{2}$ animals with increased apoptotic germ cell corpses were identified with the vital dye acridine orange (AO; Sigma) (Lettre et al. 2004). Two independent alleles (op212 and op216) were isolated from a screen of 45,000 genomes. To eliminate possible additional mutations, each mu- tant was crossed back to the wild type eight times before subsequent analysis. The ep312 deletion allele was isolated in a separate screen, performed to identify mutants involved in cep1-independent germ cell apoptosis (M. Costa, pers. comm.).

\section{Cloning of gla-3}

The op212 allele of gla-3 was mapped near the middle of Chromosome I by two-factor mapping using the marker $d p y-5$. To refine the position of $g 1 a-3$, three-factor mapping was conducted using the following marker combinations: $d p y-5$ unc-87, $d p y-5$ unc-29, and dpy-24 unc-75. This analysis placed gla-3 closer to unc-29 than dpy-5. SNP mapping refined the region to $0.36 \mathrm{cM}$ (roughly $270 \mathrm{~kb}$ ), which included 40 predicted genes. We then performed RNAi feeding of the 40 genes in this interval. Only inactivation of T02E1.3 resulted in increased germline apoptosis. The molecular changes induced by the op212, op216, and ep312 mutations were determined by PCR amplification of the gla-3 locus from the respective mutants, followed by sequencing of both strands of the amplification product.

\section{RNA interference}

Feeding RNAi was performed as previously described (Lettre et al. 2004). Feeding RNAi constructs were obtained either from the Ahringer library (Kamath et al. 2003) or from the ORFeome RNAi collection (Rual et al. 2004), or were generated by subcloning from cDNAs (for bir-1 and $g f p$ ).

\section{Germline apoptosis}

Apoptotic germ cell corpses were identified and quantitated based on their characteristic morphology under differential interference contrast (DIC) optics, as previously described (Gumienny et al. 1999).

\section{Brood size and oocyte counts}

Brood size counts were performed as previously described (Hofmann et al. 2002). Oocyte counts were performed according to Hajnal and Berset (2002).

\section{Northern analysis}

Total RNA was isolated using TRIZOL reagent (GIBCO-BRL) from cultures of synchronized wild-type worms at different developmental stages as well as from g1a-3(op212), glp-4(bn2ts), fem-2(e2105ts), and fem-3(q20sd) adults. Poly(A) $)^{+}$selection of mRNAs from staged adults was performed using the MicroPolyA kit (Ambion). RNA samples were subjected to formaldehyde agarose gel electrophoresis, transferred to Hybond N+ nylon membrane, and probed with a full-length gla-3 cDNA amplified from plasmid yk6h11 and labeled with ${ }^{32} \mathrm{P}$ using the random hexamer primer method.

\section{Generation of GLA-3 antibodies}

Rabbit polyclonal antibody T02E1.3a (1301) was raised against the TO2E1.3a peptide KTQEISVVIDPRDA, whereas antibody T02E1.3b (1297) was raised against the T02E1.3b peptide LLNSDMDPVRNLES. Peptides were synthesized (Sigma-Genosys) and injected into two rabbits according to Sigma protocols.

\section{Western blot analysis}

Adult worms were washed off the plates with water. Worm pellets were frozen in liquid nitrogen and kept at $-80^{\circ} \mathrm{C}$. To 
extract proteins, an equal volume of acid-washed glass beads (Sigma) was added to the frozen worm pellet, and the tubes were put in a bead beater (FastPrep FP120) for $35 \mathrm{sec}$ at speed 6.5. Following addition of $100-300 \mu \mathrm{L}$ of RIPA buffer $(50 \mathrm{mM}$ Tris at $\mathrm{pH}$ 8.0, $150 \mathrm{mM} \mathrm{NaCl}, 1 \% \mathrm{NP}-40,1 \%$ deoxycholate, 1\% SDS, 1 tablet/10 mL Complete Mini protease inhibitor [Roche]) or immunoprecipitation buffer $(25 \mathrm{mM}$ HEPES-NaOH at $\mathrm{pH} 7.4$, $150 \mathrm{mM} \mathrm{NaCl}, 0.2 \mathrm{mM}$ DTT, 10\% glycerol, $1 \%$ Triton X-100, 1 tablet/10 mL Complete Mini protease inhibitor [(Roche]), tubes were vortexed and incubated on ice for $10 \mathrm{~min}$. Extracts were centrifuged for $15 \mathrm{~min}$ at $13,000 \mathrm{rpm}$ at $4^{\circ} \mathrm{C}$. Supernatants were collected and kept at $-80^{\circ} \mathrm{C}$. Protein quantification was done using the Bio-Rad protein assay as recommended, with bovine serum albumin (BSA) as standard. The antibody dilutions used were 1:1000 for GLA-3 antibodies and 1:10,000 for $\alpha$-tubulin (Upstate). Secondary HRP-labeled anti-rabbit and anti-mouse antibodies (Sigma) were used at 1:100,000 and 1:40,000, respectively, and detected using ECL (Sigma).

\section{Transgenic worms}

The $P_{\text {gla-3a }}:: 2 x N L S::$ GFP unc-119(+) construct (pEK1) was bombarded into unc-119(ed3) worms as previously described (Praitis et al. 2001; Hofmann et al. 2002). Integration of each construct was determined by loss of visible Unc-119 offspring. At least three integrated lines were generated; all showed the same expression pattern.

\section{Reverse two-hybrid selection}

The system we used is a variation of the original reverse twohybrid protocol developed by Vidal (Vidal et al. 1996a,b). Briefly, the mpk-1 wild-type ORF was mutagenized by PCR using 30 amplification cycles and Platinum Taq DNA polymerase (Invitrogen). To build the mpk-1 mutant library, the gel-purified PCR product was cloned into the pDONR-Express vector using the Gateway in vitro cloning system (Invitrogen). In this vector, the ORF is cloned under control of an IPTG-inducible promoter in frame with the $\mathrm{Kan}^{R}$ gene. Clones containing PCR-induced STOP mutations are removed by plating TOP-10 (Invitrogen) bacteria transformants on IPTG $(1 \mathrm{mM})$ and $\mathrm{Kan}(80 \mu \mathrm{g} / \mathrm{mL})$ selective medium. About 500,000 independent transformants were obtained. The ORFs were LR cloned into the pPC-97 Gal-4 DNA-binding domain yeast two-hybrid vector and retransformed into electrocompetent TOP-10 cells. This library was transformed into MaV203 yeast cells containing GLA-3 fused to the Gal-4 activation domain. Cotransformed cells were selected for their ability to grow on -Leu, -Trp, +5-FOA (5-fluoro-orotic acid) medium, and the mpk-1 ORF was amplified by PCR and sequenced so that the causative mutation could be identified. These mpk-1 mutants were retested by gap repair in fresh MaV203 containing the AD GLA-3 fusion, to confirm that the interaction had been disrupted because of the mutation (Walhout and Vidal 2001).

\section{Visualization of amino acid changes on MPK-1}

Mutations identified in mpk-1 using the yeast reverse two-hybrid system were mapped to the structure model of MPK-1 (SWISS-PROT P39745), which was derived from the rat ERK2 (SWISS-PROT P63086) crystal structure (Zhang et al. 1993, 1994). The location of the changed amino acids was visualized on the 3D structure using Swiss-PdbViewer (Guex and Peitsch 1997).

\section{GST pull-downs}

mpk-1a or mpk-1b ORFs were cloned into pDEST-27, which contains GST coding sequence upstream of the Gateway recom- bination site (Invitrogen). gla-3a and gla-3b ORFs were cloned into pDEST-CMV-myc, which contains the myc tag upstream of the Gateway recombination site (Invitrogen). Both vectors use the CMV promoter to drive expression of the fusion protein. Plasmids were transfected into 293T cells using Lipofectamin 2000 reagent according to the manufacturer's instructions (Invitrogen). Cells were cultured for $2 \mathrm{~d}$ in DMEM medium, and lysed in $0.1 \%$ NP-40 buffer $(50 \mathrm{mM}$ Tris- $\mathrm{HCl}$ at $\mathrm{pH} 7.5,150 \mathrm{mM}$ $\mathrm{NaCl}, 1 \mathrm{mM}$ EDTA, and complete protease inhibitors [Amersham]). Lysates were centrifuged at $14,000 \mathrm{~g}$, before purification of protein complexes using glutathione Sepharose beads. Purified complexes and control lysate samples were run on $\mathrm{Nu}-$ PAGE acrylamide gels (Invitrogen), and Myc- and GST-tagged proteins were detected using standard immunoblotting techniques. Mouse monoclonal anti-Myc (clone 9E10) and rabbit polyclonal anti-GST were purchased from Sigma.

\section{Immunofluorescence and imaging}

Immunostaining was performed essentially as described (Page et al. 2001). Briefly, dissected gonads were fixed in $1 \%$ paraformaldehyde in PBS and permeabilized in TBS supplemented with $0.1 \%$ Triton X-100. The samples were subsequently incubated with the following antibodies: rabbit anti-GLA-3A 1301, 1:1000 or rabbit anti-GLA-3B $1297,1: 1000$ in $5 \%$ BSA in TBS, at $4^{\circ} \mathrm{C}$ overnight. Anti-rabbit AlexaFluor 594 or anti-mouse AlexaFluor 594 IgG antibodies were used as secondary antibodies (Molecular Probes). DAPI was added to a final concentration of $200 \mathrm{ng} / \mathrm{mL}$, and samples were mounted for microscopy. All images were analyzed by light microscopy with a Zeiss Axioskop equipped with epifluorescence and differential interference contrast (DIC) optics. Digital images were acquired and processed using a CCD camera and Openlab software. Histochemical staining of $\beta$-galactosidase with 5-bromo-4-chloro-3-indolyl- $\beta$ D-galactopyranoside (X-Gal) was performed as described (Zdinak et al. 1997).

\section{Acknowledgments}

We thank the C. elegans Gene Knockout Consortium and the Caenorhabditis Genetics Center for providing strains; M. Costa for the gla-3(ep312) allele; L. Broday for the developmental Northern blot; Y. Kohara for plasmid yk6h11; and A. Hajnal, M. Boxem, D. Hill, and T. Berset for discussions and comments. This work was supported by grants from the Swiss National Science Foundation, the Josef Steiner Foundation, the European Union (FP5 project Apoclear), and the Ernst Hadorn Foundation. P.-O.V. was supported by an integrated interactome mapping grant (NCI/NIH 1 R33 CA105405-01), and S.M. was supported by generation of a C. elegans protein interaction database grant (NHGRI/NIH 5 R01 HG01715-07) and training grant (NCI/NIH 5 T32 CA09361-25).

\section{References}

Ahmed, S., Alpi, A., Hengartner, M.O., and Gartner, A. 2001. C. elegans RAD-5/CLK-2 defines a new DNA damage checkpoint protein. Curr. Biol. 11: 1934-1944.

Alessi, D.R., Cohen, P., Ashworth, A., Cowley, S., Leevers, S.J., and Marshall, C.J. 1995. Assay and expression of mitogenactivated protein kinase, MAP kinase kinase, and Raf. Methods Enzymol. 255: 279-290.

Bai, C. and Tolias, P.P. 1996. Cleavage of RNA hairpins mediated by a developmentally regulated $\mathrm{CCCH}$ zinc finger protein. Mol. Cell. Biol. 16: 6661-6667. 
Barabino, S.M., Hubner, W., Jenny, A., Minvielle-Sebastia, L., and Keller, W. 1997. The $30-\mathrm{kD}$ subunit of mammalian cleavage and polyadenylation specificity factor and its yeast homolog are RNA-binding zinc finger proteins. Genes \& Dev. 11: 1703-1716.

Barton, M.K., Schedl, T.B., and Kimble, J. 1987. Gain-of-function mutations of fem-3, a sex-determination gene in Caenorhabditis elegans. Genetics 115: 107-119.

Beanan, M.J. and Strome, S. 1992. Characterization of a germline proliferation mutation in C. elegans. Development 116: 755-766.

Bender, C.F., Sikes, M.L., Sullivan, R., Huye, L.E., Le Beau, M.M., Roth, D.B., Mirzoeva, O.K., Oltz, E.M., and Petrini, J.H. 2002. Cancer predisposition and hematopoietic failure in Rad50(S/S) mice. Genes \& Dev. 16: 2237-2251.

Berset, T., Hoier, E.F., Battu, G., Canevascini, S., and Hajnal, A. 2001. Notch inhibition of RAS signaling through MAP kinase phosphatase LIP-1 during C. elegans vulval development. Science 291: 1055-1058.

Boag, P.R., Nakamura, A., and Blackwell, T.K. 2005. A conserved RNA-protein complex component involved in physiological germline apoptosis regulation in C. elegans. Development 132: 4975-4986.

Bolanowski, M.A., Russell, R.L., and Jacobson, L.A. 1981. Quantitative measures of aging in the nematode Caenorhabditis elegans. I. Population and longitudinal studies of two behavioral parameters. Mech. Ageing Dev. 15: 279-295.

Brenner, S. 1974. The genetics of Caenorhabditis elegans. Genetics 77: 71-94.

Cao, H., Dzineku, F., and Blackshear, P.J. 2003. Expression and purification of recombinant tristetraprolin that can bind to tumor necrosis factor- $\alpha$ mRNA and serve as a substrate for mitogen-activated protein kinases. Arch. Biochem. Biophys. 412: 106-120.

Carballo, E., Lai, W.S., and Blackshear, P.J. 2000. Evidence that tristetraprolin is a physiological regulator of granulocytemacrophage colony-stimulating factor messenger RNA deadenylation and stability. Blood 95: 1891-1899.

Chen, F., Hersh, B.M., Conradt, B., Zhou, Z., Riemer, D., Gruenbaum, Y., and Horvitz, H.R. 2000. Translocation of $C$. elegans CED-4 to nuclear membranes during programmed cell death. Science 287: 1485-1489.

Chrestensen, C.A., Schroeder, M.J., Shabanowitz, J., Hunt, D.F., Pelo, J.W., Worthington, M.T., and Sturgill, T.W. 2004. MAPKAP kinase 2 phosphorylates tristetraprolin on in vivo sites including Ser178, a site required for 14-3-3 binding. J. Biol. Chem. 279: 10176-10184.

Church, D.L., Guan, K.L., and Lambie, E.J. 1995. Three genes of the MAP kinase cascade, mek-2, mpk-1/sur-1 and let-60 ras, are required for meiotic cell cycle progression in Caenorhabditis elegans. Development 121: 2525-2535.

Derry, W.B., Putzke, A.P., and Rothman, J.H. 2001. Caenorhabditis elegans p53: Role in apoptosis, meiosis, and stress resistance. Science 294: 591-595.

Detwiler, M.R., Reuben, M., Li, X., Rogers, E., and Lin, R. 2001. Two zinc finger proteins, OMA-1 and OMA-2, are redundantly required for oocyte maturation in C. elegans. Dev. Cell 1: 187-199.

DuBois, R.N., McLane, M.W., Ryder, K., Lau, L.F., and Nathans, D. 1990. A growth factor-inducible nuclear protein with a novel cysteine/histidine repetitive sequence. J. Biol. Chem. 265: 19185-19191.

Gartner, A., Milstein, S., Ahmed, S., Hodgkin, J., and Hengartner, M.O. 2000. A conserved checkpoint pathway mediates DNA damage-induced apoptosis and cell cycle arrest in $C$. elegans. Mol. Cell 5: 435-443.
Guedes, S. and Priess, J.R. 1997. The C. elegans MEX-1 protein is present in germline blastomeres and is a P granule component. Development 124: 731-739.

Guex, N. and Peitsch, M.C. 1997. SWISS-MODEL and the Swiss-PdbViewer: An environment for comparative protein modeling. Electrophoresis 18: 2714-2723.

Gumienny, T.L., Lambie, E., Hartwieg, E., Horvitz, H.R., and Hengartner, M.O. 1999. Genetic control of programmed cell death in the Caenorhabditis elegans hermaphrodite germline. Development 126: 1011-1022.

Hajnal, A. and Berset, T. 2002. The C. elegans MAPK phosphatase LIP-1 is required for the $\mathrm{G}_{2} / \mathrm{M}$ meiotic arrest of developing oocytes. EMBO J. 21: 4317-4326.

Hengartner, M.O. 1997. Apoptosis and the shape of death. Dev. Genet. 21: 245-248.

Hofmann, E.R., Milstein, S., Boulton, S.J., Ye, M., Hofmann, J.J., Stergiou, L., Gartner, A., Vidal, M., and Hengartner, M.O. 2002. Caenorhabditis elegans HUS-1 is a DNA damage checkpoint protein required for genome stability and EGL1-mediated apoptosis. Curr. Biol. 12: 1908-1918.

Hubbard, E.J. and Greenstein, D. 2000. The Caenorhabditis elegans gonad: A test tube for cell and developmental biology. Dev. Dyn. 218: 2-22.

Kamath, R.S., Fraser, A.G., Dong, Y., Poulin, G., Durbin, R., Gotta, M., Kanapin, A., Le Bot, N., Moreno, S., Sohrmann, M., et al. 2003. Systematic functional analysis of the Caenorhabditis elegans genome using RNAi. Nature 421: 231237.

Karashima, T., Sugimoto, A., and Yamamoto, M. 2000. Caenorhabditis elegans homologue of the human azoospermia factor DAZ is required for oogenesis but not for spermatogenesis. Development 127: 1069-1079.

Kim, M.R. and Tilly, J.L. 2004. Current concepts in Bcl-2 family member regulation of female germ cell development and survival. Biochim. Biophys. Acta 1644: 205-210.

Kinchen, J.M., Cabello, J., Klingele, D., Wong, K., Feichtinger, R., Schnabel, H., Schnabel, R., and Hengartner, M.O. 2005. Two pathways converge at CED-10 to mediate actin rearrangement and corpse removal in C. elegans. Nature 434: 93-99.

Lackner, M.R. and Kim, S.K. 1998. Genetic analysis of the Caenorhabditis elegans MAP kinase gene mpk-1. Genetics 150: 103-117.

Lai, W.S., Carballo, E., Strum, J.R., Kennington, E.A., Phillips, R.S., and Blackshear, P.J. 1999. Evidence that tristetraprolin binds to AU-rich elements and promotes the deadenylation and destabilization of tumor necrosis factor $\alpha$ mRNA. Mol. Cell. Biol. 19: 4311-4323.

Lai, W.S., Carballo, E., Thorn, J.M., Kennington, E.A., and Blackshear, P.J. 2000. Interactions of $\mathrm{CCCH}$ zinc finger proteins with mRNA. Binding of tristetraprolin-related zinc finger proteins to Au-rich elements and destabilization of mRNA. J. Biol. Chem. 275: 17827-17837.

Lettre, G. and Hengartner, M.O. 2006. Developmental apoptosis in C. elegans: A complex CEDnario. Nat. Rev. Mol. Cell Biol. 7: 97-108.

Lettre, G., Kritikou, E.A., Jaeggi, M., Calixto, A., Fraser, A.G., Kamath, R.S., Ahringer, J., and Hengartner, M.O. 2004. Genome-wide RNAi identifies p53-dependent and -independent regulators of germ cell apoptosis in C. elegans. Cell Death Differ. 11: 1198-1203.

Li, S., Armstrong, C.M., Bertin, N., Ge, H., Milstein, S., Boxem, M., Vidalain, P.O., Han, J.D., Chesneau, A., Hao, T., et al. 2004. A map of the interactome network of the metazoan C. elegans. Science 303: 540-543.

Lim, D.S. and Hasty, P. 1996. A mutation in mouse rad51 re- 
sults in an early embryonic lethal that is suppressed by a mutation in p53. Mol. Cell. Biol. 16: 7133-7143.

Mahtani, K.R., Brook, M., Dean, J.L., Sully, G., Saklatvala, J., and Clark, A.R. 2001. Mitogen-activated protein kinase p38 controls the expression and posttranslational modification of tristetraprolin, a regulator of tumor necrosis factor $\alpha$ mRNA stability. Mol. Cell. Biol. 21: 6461-6469.

Mello, C.C., Schubert, C., Draper, B., Zhang, W., Lobel, R., and Priess, J.R. 1996. The PIE-1 protein and germline specification in C. elegans embryos. Nature 382: 710-712.

Miller, M.A., Nguyen, V.Q., Lee, M.H., Kosinski, M., Schedl, T., Caprioli, R.M., and Greenstein, D. 2001. A sperm cytoskeletal protein that signals oocyte meiotic maturation and ovulation. Science 291: 2144-2147.

Moghal, N. and Sternberg, P.W. 2003. The epidermal growth factor system in Caenorhabditis elegans. Exp. Cell Res. 284: 150-159.

Navarro, R.E., Shim, E.Y., Kohara, Y., Singson, A., and Blackwell, T.K. 2001. cgh-1, a conserved predicted RNA helicase required for gametogenesis and protection from physiological germline apoptosis in C. elegans. Development 128: 3221-3232.

Page, B.D., Guedes, S., Waring, D., and Priess, J.R. 2001. The C. elegans E2F- and DP-related proteins are required for embryonic asymmetry and negatively regulate Ras/MAPK signaling. Mol. Cell 7: 451-460.

Praitis, V., Casey, E., Collar, D., and Austin, J. 2001. Creation of low-copy integrated transgenic lines in Caenorhabditis elegans. Genetics 157: 1217-1226.

Rual, J.F., Ceron, J., Koreth, J., Hao, T., Nicot, A.S., HirozaneKishikawa, T., Vandenhaute, J., Orkin, S.H., Hill, D.E., van den Heuvel, S., et al. 2004. Toward improving Caenorhabditis elegans phenome mapping with an ORFeome-based RNAi library. Genome Res. 14: 2162-2168.

Schneider, S.Q. and Bowerman, B. 2003. Cell polarity and the cytoskeleton in the Caenorhabditis elegans zygote. Annu. Rev. Genet. 37: 221-249.

Schubert, C.M., Lin, R., de Vries, C.J., Plasterk, R.H., and Priess, J.R. 2000. MEX-5 and MEX-6 function to establish soma/ germline asymmetry in early C. elegans embryos. Mol. Cell 5: 671-682.

Schumacher, B., Hofmann, K., Boulton, S., and Gartner, A. 2001. The C. elegans homolog of the p53 tumor suppressor is required for DNA damage-induced apoptosis. Curr. Biol. 11: $1722-1727$.

Seydoux, G. and Schedl, T. 2001. The germline in C. elegans: Origins, proliferation, and silencing. Int. Rev. Cytol. 203: 139-185.

Shimada, M., Kawahara, H., and Doi, H. 2002. Novel family of $\mathrm{CCCH}$-type zinc-finger proteins, MOE-1, -2 and -3, participates in C. elegans oocyte maturation. Genes Cells 7: 933 947.

Sugiura, R., Kita, A., Shimizu, Y., Shuntoh, H., Sio, S.O., and Kuno, T. 2003. Feedback regulation of MAPK signalling by an RNA-binding protein. Nature 424: 961-965.

Szewczyk, N.J., Hartman, J.J., Barmada, S.J., and Jacobson, L.A. 2000. Genetic defects in acetylcholine signalling promote protein degradation in muscle cells of Caenorhabditis elegans. J. Cell Sci. 113: 2003-2010.

Szewczyk, N.J., Peterson, B.K., and Jacobson, L.A. 2002. Activation of Ras and the mitogen-activated protein kinase pathway promotes protein degradation in muscle cells of Caenorhabditis elegans. Mol. Cell. Biol. 22: 4181-4188.

Tabara, H., Hill, R.J., Mello, C.C., Priess, J.R., and Kohara, Y. 1999. pos-1 encodes a cytoplasmic zinc-finger protein essential for germline specification in C. elegans. Development
126: $1-11$.

Tanoue, T. and Nishida, E. 2002. Docking interactions in the mitogen-activated protein kinase cascades. Pharmacol. Ther. 93: 193-202.

Taylor, G.A., Thompson, M.J., Lai, W.S., and Blackshear, P.J. 1995. Phosphorylation of tristetraprolin, a potential zinc finger transcription factor, by mitogen stimulation in intact cells and by mitogen-activated protein kinase in vitro. $J$. Biol. Chem. 270: 13341-13347.

. 1996. Mitogens stimulate the rapid nuclear to cytosolic translocation of tristetraprolin, a potential zinc-finger transcription factor. Mol. Endocrinol. 10: 140-146.

Tilly, J.L. 2001. Commuting the death sentence: How oocytes strive to survive. Nat. Rev. Mol. Cell Biol. 2: 838-848.

Varnum, B.C., Lim, R.W., Sukhatme, V.P., and Herschman, H.R. 1989. Nucleotide sequence of a cDNA encoding TIS11, a message induced in Swiss 3T3 cells by the tumor promoter tetradecanoyl phorbol acetate. Oncogene 4: 119-120.

Vidal, M., Brachmann, R.K., Fattaey, A., Harlow, E., and Boeke, J.D. 1996a. Reverse two-hybrid and one-hybrid systems to detect dissociation of protein-protein and DNA-protein interactions. Proc. Natl. Acad. Sci. 93: 10315-10320.

Vidal, M., Braun, P., Chen, E., Boeke, J.D., and Harlow, E. 1996b. Genetic characterization of a mammalian protein-protein interaction domain by using a yeast reverse two-hybrid system. Proc. Natl. Acad. Sci. 93: 10321-10326.

Walhout, A.J. and Vidal, M. 2001. High-throughput yeast twohybrid assays for large-scale protein interaction mapping. Methods 24: 297-306.

Weischenfeldt, J., Lykke-Andersen, J., and Porse, B. 2005. Messenger RNA surveillance: Neutralizing natural nonsense. Curr. Biol. 15: R559-R562.

Worthington, M.T., Pelo, J.W., Sachedina, M.A., Applegate, J.L., Arseneau, K.O., and Pizarro, T.T. 2002. RNA binding properties of the AU-rich element-binding recombinant Nup475/ TIS11/tristetraprolin protein. J. Biol. Chem. 277: 48558-48564.

Yan, N., Gu, L., Kokel, D., Chai, J., Li, W., Han, A., Chen, L., Xue, D., and Shi, Y. 2004. Structural, biochemical, and functional analyses of CED-9 recognition by the proapoptotic proteins EGL-1 and CED-4. Mol. Cell 15: 999-1006.

Yan, N., Chai, J., Lee, E.S., Gu, L., Liu, Q., He, J., Wu, J.W., Kokel, D., Li, H., Hao, Q., et al. 2005. Structure of the CED4-CED-9 complex provides insights into programmed cell death in Caenorhabditis elegans. Nature 437: 831-837.

Zdinak, L.A., Greenberg, I.B., Szewczyk, N.J., Barmada, S.J., Cardamone-Rayner, M., Hartman, J.J., and Jacobson, L.A. 1997. Transgene-coded chimeric proteins as reporters of intracellular proteolysis: Starvation-induced catabolism of a lacZ fusion protein in muscle cells of Caenorhabditis elegans. J. Cell. Biochem. 67: 143-153.

Zhang, F., Robbins, D.J., Cobb, M.H., and Goldsmith, E.J. 1993. Crystallization and preliminary X-ray studies of extracellular signal-regulated kinase-2/MAP kinase with an incorporated His-tag. J. Mol. Biol. 233: 550-552.

Zhang, F., Strand, A., Robbins, D., Cobb, M.H., and Goldsmith, E.J. 1994. Atomic structure of the MAP kinase ERK2 at $2.3 \AA$ resolution. Nature 367: 704-711.

Zhang, J., Zhou, B., Zheng, C.F., and Zhang, Z.Y. 2003. A bipartite mechanism for ERK2 recognition by its cognate regulators and substrates. J. Biol. Chem. 278: 29901-29912. 


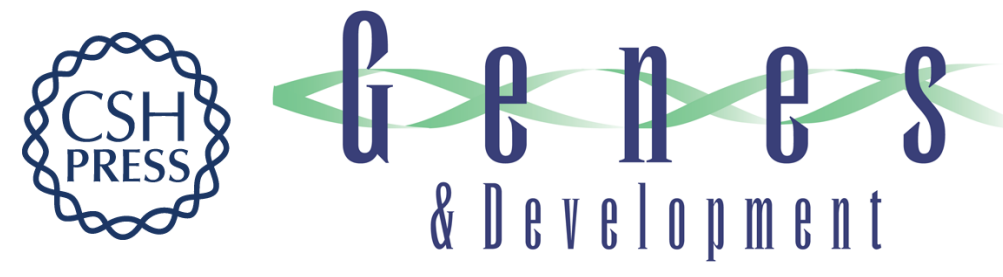

\section{C. elegans GLA-3 is a novel component of the MAP kinase MPK-1 signaling pathway required for germ cell survival}

Ekaterini A. Kritikou, Stuart Milstein, Pierre-Olivier Vidalain, et al.

Genes Dev. 2006, 20:

Access the most recent version at doi:10.1101/gad.384506

Supplemental http://genesdev.cshlp.org/content/suppl/2006/08/18/20.16.2279.DC1
Material

References This article cites 71 articles, 35 of which can be accessed free at:

http://genesdev.cshlp.org/content/20/16/2279.full.html\#ref-list-1

License

Email Alerting

Receive free email alerts when new articles cite this article - sign up in the box at the top

Service

right corner of the article or click here.

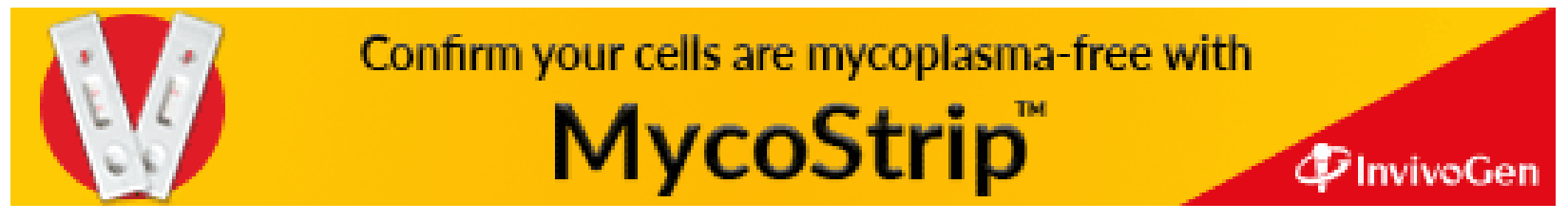

